Relations industrielles

Industrial Relations

\title{
Nos responsabilités chrétiennes en face du chômage
}

\section{Paul-Émile Léger}

Volume 14, numéro 1, janvier 1959

URI : https://id.erudit.org/iderudit/1022348ar

DOI : https://doi.org/10.7202/1022348ar

Aller au sommaire du numéro

Éditeur(s)

Département des relations industrielles de l'Université Laval

ISSN

0034-379X (imprimé)

1703-8138 (numérique)

Découvrir la revue

Citer ce document

Léger, P.-É. (1959). Nos responsabilités chrétiennes en face du chômage. Relations industrielles / Industrial Relations, 14(1), 126-140.

https://doi.org/10.7202/1022348ar

Tous droits réservés @ C Département des relations industrielles de l’Université Laval, 1959
Ce document est protégé par la loi sur le droit d'auteur. L’utilisation des services d'Érudit (y compris la reproduction) est assujettie à sa politique d'utilisation que vous pouvez consulter en ligne.

https://apropos.erudit.org/fr/usagers/politique-dutilisation/ 


\section{NOS RESPONSABILITES CHRETIENNES EN FACE DU CHOMAGE}

\section{S.E. le Cardinal Paul-Emile Lécer}

Qui ne connait cet émouvant récit évangélique de la multiplication des pains? Le divin Maître, en présence d'une multitude épuisée, exposée à périr de faim, appelle ses disciples et leur confie son inquiétude: «J'ai compassion pour cette foule, dit-Il... ils n'ont rien à manger! » (Matt. XV, 32.)

Il y a dans cette touchante préoccupation du Christ, en présence de la misère d'une foule, tout un programme d'action. Nous pouvons y retracer un triple enseignement:

—d'un regard compatissant, Jésus mesure toute l'étendue de la souffrance de ce peuple et en prévoit toutes les conséquences;

-mais Il ne se contente pas de constater la misère, Il oherche aussitôt les moyens de la soulager. Il fait ramasser quelques pains et quelques poissons, les bénit et les fait distribuer;

enfin, nous apprend l'Evangile, son intervention a été pratique puisque « tous mangèrent et furent rassasiés ». (Marc, VIII, 8.)

Chers Diocésains, au début de cette Lettre pastorale où Nous voulons vous rappeler les responsabilités du chrétien en face du terrible fléau du chômage, Nous formons les voeux les plus ardents pour que l'action de tous s'inspire de la triple Jeçon contenue dans ce récit.

-Un chrétien doit d'abord se sentir solidaire de la misère et de la souffrance de ses frères;

-ensuite, loin de s'attarder à des récriminations inutiles, le chrétien authentique est poussé par la charité à rechencher les moyens les plus efficaces de remédier au désordre social;

-enfin, il est convaincu qu'une action sociale, éclairée et généreuse, entreprise par amour de Dieu et du prochain, ne manque pas de produire des résultats heureux.

\section{PREMIERE PARTIE}

\section{La situation actuelle et les éléments d'une solution juste et durable}

\section{- I - \\ Gravité de la situation actuelle}

Depuis quelques années, la plaie du chômage affecte notre société d'une façon persistante et ne cesse de s'aggraver. Ce mal afflige un très grand nombre de travailleurs, plonge les familles dans la misère et compromet dangereusement l'ordre social.

On Nous informe qu'il existe au-delà de 80,000 chômeurs à Montréal; et si Nous considérons que chaque chômeur est responsable de la subsistance de deux 
autres personnes, en moyenne, Nous devons conclure qu'environ 240,000 persomes sont victimes de ce fléau.

Nous mesurerons davantage la gravité de la situation si Nous songeons qu'il se trouve parmi eux de 10 à 15,000 jeunes chômeurs. Ces jeunes se présentent à la société avec leur dynamisme, leurs espoirs légitimes et leur volonté de travailler et se voient malheureusement refuser les emplois qu'ils convoitent.

Qui oserait alors prétendre, devant cette situation, que tout genre de chômage est normal et constitue tout au plus un «mal nécessaire »? Une telle prétention serait aussi peu d'accord avec la morale qu'avec la science actuelle. Le chrétien ne peut rester indifférent devant une situation qui engendre des conséquences aussi douloureuses. Au-delà des statistiques et des chiffres, il faut voir des hommes et des femmes, des enfants et des vieillards, réduits à lindigence et exposés aux terribles tentations du désespoir.

\section{TERRIBLES CONSÉQuenCES}

En effet, les conséquences du chômage sont graves et multiples. Les nombreux chômeurs «qui ne demandent rien d'autre que de gagner honorablement leur pain quotidien, que le divin commandement leur fait sollioiter chaque jour du Père céleste» sont empêchés de gagner leur vie et celle de leur famille (Pie XI, 2 octobre 1931).

Privés de tous les avantages moraux assurés par le travail, les chômeurs ont limpression d'être inutiles ou de trop dans la société. Cette situation bouleverse la famille et en compromet l'harmonie. Le père de famille est humilié de ne pouvoir s'acquitter de ses responsabilités envers les siens; il se sent diminué et déchu. Le découragement peut s'emparer de lui et le désoeuvrement l'expose à toutes sortes de tentations. La criminalité qui augmente considérablement en période de chômage intense souligne encore davantage la gravité de ce mal.

«Si un homme souffre du chômage pour une longue période, précise un économiste, des réactions morbides affecteront presque certainement sa qualité d'homme et d'ouvrier. Ce n'est pas seulement que son habileté technique diminue par manque de pratique. L'habitude de travailler se perdra, le respect de soi-même et la confiance en soi peuvent se détruire et, quand l'oocasion se présente à nouveau, cet homme qui n'était jusque là qu'un «inemployé », se trouvera devenu «inemployable». Pendant ce temps, sa vie privée peut s'être écroulée et l'atmosphère dans laquelle ses enfants grandissent peut avoir été empoisonnée».

Si les théories du plein emploi restent discutées entre économistes, le chômage n'est plus considéré par eux comme une nécessité de nature.

Le chòmage compromet gravement l'ordre social puisqu'il est susceptible d'engendrer chez ses victimes le mécontentement et lamertume envers une société qui se montre incapable d'utiliser les forces vives qui ne demandent qu'à être employées. L'expérience de pays étrangers nous prouve que les fauteurs de désondre, les démagogues, y trouvent toujours un terrain de choix pour encourager la lutte des classes. A cause de l'instabilité dont il est le résultat, le chômage devient un des pires destructeurs de l'humanité. 
En appauvrissant ses victimes, le chômage diminue la consommation, provoque la stagnation économique dans d'autres branches de l'économie et paralyse le progrès. Non seulement la société ne profite pas des ressources en travail, véritablement stérilisées, mais elle doit de plus subvenir aux besoins des chômeurs.

\section{Triste PARADOXE}

Comment nous réconcilier avec le tableau paradoxal qui présente des milliers de citoyens sans travail dans une contrée immensément riche et en plein développement.

Notre pays, pouvions-nous lire dans un récent rapport sur les Perspectives Economiques du Canada, «est un des rares pays qui trouvent chez eux la plupart des matières premières nécessaires à toute grande puissance industrielle, et depuis. 1945, une foule de découvertes et de réalisations sont venues le mettre davantage en mesure de se suffire... Parmi les autres nations du monde, le Canada peut se compter particulièrement avantagé et heureux». (Perspectives Economiques du Canada, pp. 17 et 34.)

La richesse publique vient moins de l'abondance des biens que de leur juste répartition. Un peuple qui ne bénéficierait pas d'une juste distribution de richesses matérielles même surabondantes, serait un peuple pauvre dans un pays riche.

Nous ne pouvons cacher les préoccupations que Nous a inspirées le chômage considérable qui a sévi l'hiver dernier et qui atteint malheureusement à l'heure présente tant de foyers ouvriers.

Les travailleurs qui ont épuisé leurs prestations d'assurance-chômage ont été plongés dans une situation intolérable. Ceux qui ont repris le travail mais qui ne pourront travailler assez longtemps pour être éligibles aux prestations se trouveront dans la même situation s'ils perdent leur emploi actuel.

Par ailleurs, un bon nombre d'ouvriers qui ont un emploi vivent dans la crainte constante de le perdre.

Une situation qui engendre la misère d'un trop grand nombre de familles et qui cause une telle insécurité exige de nous tous un examen sérieux du régime économique dans lequel nous vivons.

\section{$-\mathrm{II}-$ \\ L'intervention de I'Eglise}

Croyez-vous, Nos très chers frères, qu'un pasteur d'âmes puisse rester plus longtemps silencieux devant un tel état de chose, quand il voit des milliers et des milliers de ses diocésains souffrir, matériellement et moralement, placés dans des conditions sociales qui rendent ardue une conduite conforme aux préceptes chrétiens?

Evidemment, il ne Nous appartient pas de proposer des solutions techniques au chômage. Et Nous Nous garderons bien d'entrer dans l'analyse détaillée d'un problème aussi complexe. Le rôle de la hiérarchie ecclésiastique d'ailleurs n'est pas de se substituer aux employeurs, aux économistes, aux dirigeants ouvriers, ni aux 
hommes d'Etat. Il appartient à eux seuls de choisir, parmi les divers moyens concevables, ceux qui sont les plus ungents et les plus rentables du point de vue humain.

\section{MOTIFS DE SON INTERVENTION}

Le droit et le devoir qu'a l'Eglise d'intervenir dans le domaine économicosocial reposent à la fois, d'une part, sur la fin de l'Eglise qui est de sauver les âmes et, d'autre part, sur la fin même de l'organisme économique et social «qui est de procurer à ses membres tous les biens que les ressources de la nature et de l'industrie, ainsi qu'une organisation saine de la vie économique, ont le moyen de leur procurer 》 (Pie XII, 5-7-52) et tout cela pour qu'ils puissent mieux atteindre leur destinée et assurer leur salut.

Comme Elle est chargée d'orienter l'homme, tous les hommes vers leur fin véritable et ultime, qu'Elle est soucieuse de leur offrir les moyens d'y parvenir, vous conviendrez que l'Eglise ne peut rester indifférente aux conditions concrètes dans lesquelles se déroule leur existence. Surtout si ces circonstances risquent le moindnement d'entraver la poursuite de leur destinée.

«L'Eglise, déclarait Pie XII aux membres du Sacré-Collège (20-2-46) ne peut s'enfermer inerte dans le secret de ses temples et déserter la mission divinement providentielle de former l'homme complet, et par là de collaborer sans cesse à la réalisation d'un fondement solide de la société. Cette mission lui est essentielle».

\section{NATURE DE SON INTERVENTION}

C'est pourquoi l'Eglise, en tant que gandienne et interprète de l'ordre naturel et de la loi morale inscrite au coeur de l'homme et complétée par la Révélation, ne se lasse jamais de rappeler les principes qui doivent assurer la bonne orientation de la société et de ses membres vers leur fin propre. C'est là sa mission d'enseignement!

Mais dans le domaine économique et social où, selon l'expression même de Benoît XV (11-3-20) \&le salut éternel des âmes est bien souvent en péril », l'Eglise ne se contente pas d'intervenir par son enseignement: bien plus, Elle engage tous ses membres, laïcs et prêtres, à prendre des responsabilités dans l'action, à traduire leur credo dans les faits. Cet appel, Elle le lance tout particulièrement aux lä̈cs qui sont personnellement engagés dans les structures temporelles, pour qu'ils tâchent de les mener à leur fin propre et d'y infuser l'esprit chrétien.

Dans cette délicate question du chômage, l'Eglise se doit d'intervenir, non seulement en raison des conséquences graves qui en découlent mais encore parce qu'il est nécessaire de lui trouver une solution juste et durable.

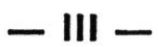

\section{Principes d'une solution juste}

Or à quelles conditions préalables peut-on espérer une juste et durable solution? A Notre avis, uniquement par le respect du droit naturel au travail et un effort pour donmer à notre vie économique une saine orientation. 


\section{A-LE DROIT AU TRAVAIL:}

\section{UN DROIT NATUREL}

Tout homme a le devoir que lui impose la nature et donc son Créateur, de conserver son existenoe et de subvenir aux besoins de sa famille. Il a donc le droit de trouver dans son travail le moyen de pourvoir à sa vie propre et à celle des siens. Ce droit ne fait en quelque sorte qu'un avec le droit de vivre.

Le chômage d'hommes valides, voulant travailler et privés de travail, est anormal et contre le droit naturel.

L'organisation sociale et économique, au contraire, en tant qu'elle est une oeuvre de raison, doit favoriser l'exercice de ce droit au travail. Une société n'est pas saine si elle ne procure pas à chacun de ses membres la possibilité de vivre humainement des fruits de son travail.

\section{LES PREMIERS RESPONSABLES}

La justice sociale demande à tous les responsables de l'économie de créer une situation économique générale qui rende possible et facile l'exercice du droit naturel de travailler. L'initiative et la responsabilité économique sont partagées entre les particuliers, leurs associations professionnelles et l'Etat. Chacun d'eux doit intervenir à sa manière, selon la fonction qu'il exerce dans l'économie.

Dans notre contexte économique il appartient particulièrement aux patrons et à leurs associations professionnelles de conserver les emplois existants et d'en créer de nouveaux.

\section{L'ACTION DE L'EtAT}

Mais le bien commun demande d'une manière beaucoup plus urgente que par le passé que l'Etat intervienne «dans la division et la distribution du travail ». Il faut donc qu'il prenne en cette matière des initiatives nouvelles et hardies, tout en respectant ce que Pie XI appelait \&la fonction supplétive de l'Etat». Il doit encourager les organismes intermédiaires à prendre les mesures qui s'imposent, il doit faciliter leur action et au besoin remédier à leurs carences.

Ce n'est que dans un tel effort commun que nous irons d̀ la conquête graduelle d'un niveau élevé et stable d'emploi. Que selon leurs responsabilités propres les entreprises privées, les pouvoirs publics et les hommes d'étude suggèrent, esquissent et réalisent de nouvelles et plus amples occasions de travail.

«... Le devoir et le droit d'organiser le travail du peuple, rappelait Pie XII, appartiennent avant tout à ceux qui y sont immédiatement intéressés: employeurs et ouvriers. Que si, ensuite, eux ne remplissent pas leur tâche, ou ne peuvent le faire par suite de spéciales circonstances extraordinaires, il rentre dans les attributions de l'Etat d'intervenir, sur ce terrain, dans la division et la distribution du travail, sous la forme et dans la mesure que demande le bien commun justement compris》(Pie XII, ler juin 1941). 
UNE POLITIQUE DE SÉCURITÉ D'EMPLOI

Une politique de sécurité d'emploi devrait nous acheminer vers une économie qui donnerait à chacun du travail et favoriserait dans la plus gnande mesure possible, la stabilité de lemploi.

Même si cette stabilité s'avère difficile, parfois même impossible dans une économie en expansion, elle n'en demeure pas moins une bien légitime aspiration de tous les travailleurs.

\& La solidarité des hommes exige non seulement au nom du sentiment fraternel mais aussi de l'avantage réciproque lui-même que l'on utilise toutes les possibilités pour conserver les emplois existants et pour en créer de nouveaux». (Pie XII, Noël 1952).

\section{B-UNE ECONOMIE SAINE}

RELATION AVEC L'ORDRE MORAL ET FINS DE L'ÉCONOMIE moral.

Une juste conception de l'économie implique une étroite relation avec l'ordre

D'une part l'homme, comme sujet agissant dans l'économie, doit régler son activité selon les exigences de la morale; d'autre part, les fins elles-mêmes de la vie économique doivent être conformes à l'ordre voulu par le Créateur.

Une économie humaine est d'abord au service de l'homme, elle est ordonnée à l'ensemble des valeurs spirituelles et matérielles qui fondent sa nature et sa dignité personnelle.

«La grande misère de l'ordre social est qu'il n'est ni profondément chrétien, ni réellement humain, mais uniquement technique et économique». (Pie XII, 31 janvier 1952). l'homme.

Une économie humaine est celle qui se règle sur les besoins primondiaux de

La fin de l'économie, a précisé le Pape Pie XII, est «d'assurer la satisfaction permanente des besoins en biens et services matériels ordonnés à leur tour à l'élévation du niveau moral, culturel et religieux» (Pie XII, Noël 1952).

On ne peut établir la véritable hiérarchie des besoins sans les référer à la loi morale. L'activité économique a donc pour but d'aménager les richesses de la création pour en faire bénéficier toutes les personnes de la collectivité.

En d'autres termes, l'économie doit exister pour l'homme et non l'homme pour l'économie. La productivité, malgré son importance capitale, ne saurait être une fin en soi.

UNE ÉCONOMIE DÉTOURNÉE DE SA FIN

Tant que nous serons dans une économie en quelque sorte détournée de son but, nous aurons à déplorer des désordres économico-sociaux tels que le chômage. 
Tant que l'économie cherchera, comme c'est malheureusement souvent le cas, la satisfaction des besoins artificiels créés par la réclame plutôt que la satisfaction des besoins primordiaux de l'homme; tant que le souci exclusif du profit pèsera de tout son poids sur la vie économique et déterminera les besoins qu'il faut satisfaire, les solutions que nous essayerons d'apporter aux maux comme le chômage demeureront incomplètes et insatisfaisantes.

Ce n'est donc pas sans raison que Notre Saint-Père le Pape portait un jugement sévère dans son message de 1956 aux exploitants agricoles: \&Il arrive trop souvent que ce ne sont plus les besoins humains qui règlent suivant leur importance naturelle et objective la vie économique et l'emploi du capital, mais au contraire le capital et ses visées de gain qui déterminent quels besoins il faut satisfaire et dans quelle mesure ils doivent l'être. En sorte que ce n'est pas le travail humain au service du bien commun qui attire et emploie le capital, mais au contraire le capital qui dispose du travail et de l'homme lui-même et qui les meut à son gré, comme les boules dans la main d'un joueur ». (Pie XII, Allocution aux agriculteurs exploitants italiens, 15 novembre 1956).

Une économie juste «doit mettre de façon stable à la portée de tous les membres de la société les conditions matérielles requises pour le développement de leur vie culturelle et spirituelle ». (Pie XII, 7 mars 1948).

Si nous ne voulons pas que le producteur soit plus longuement sacrifié à la production, il faut que celle-ci soit constamment ordonnée au bien de lhumanité.

\section{LA RÉFORME DE L'ÉCONOMIE}

La réforme de l'économie s'impose et elle est possible. Il est faux de prétendre que l'ondre économique n'obéit qu'à des mécanismes et à des lois soi-disant inéhuctables sur lesquelles l'homme ne peut avoir d'influence. La vie économique est oeuvre de raison. Sans doute a-t-elle ses lois et ses impératifs, mais par son libre comportement et son activité raisonnable, lhomme a le pouvoir de modifier le fonctionnement de l'économie et de lorienter, en tenant compte des circonstances particulières à chaque époque, en vue du bien de tous.

Lorsqu'on parle de politique économique on indique précisément l'action constructive de sujets agissants.

\section{- IV -}

\section{Les agents de cette lutte contre le chômage}

Mais un tel programme d'action, Nos Très Chers Frères, ne pourra enrayer la force centrifuge de l'individualisme, amortir les heurts et aplanir les oppositions que s'il est l'oeuvre d'une magnifique et constante collaboration de tous les agents de la vie économique et particulièrement de ceux qui s'inspirent des mêmes valeurs humaines et chrétiennes.

\section{A-LES PATRONS:}

Aux patrons il incombera avant tout de respecter les aspects moraux de l'épargne et d'assurer la constante adaptation de leur entreprise. 
L'ÉPARGNE DANS UNE ÉCONOMIE DE PROGRÈS

Puisque l'homme est sa providence il doit épargner aussitôt qu'il en a la possibilité. Mais par ailleurs dans une économie de progrès qui doit se renouveler et se développer constamment comme l'exige le bien commun, on doit dire qu'il y a généralement un devoir de justice sociale d'investir l'épargne. On ne saurait la laisser improductive.

« Ceux qui sont capables d'investir des capitaux doivent donc se demander, en considérant le bien commun, si leur conscience leur permet de ne pas faire de pareils investissements, dans les limites des possibilités économiques, dans les proportions et au moment opportun, et de se retirer à l'écart dans une vaine prudence? , (Pie XII, Noël 1952).

\section{Dynamisme et adaptation de L'entreprise}

Les patrons doivent également pour lutter contre la stagnation économique et le chômage, rivaliser d'audace, d'ingéniosité et d'efficacité.

Ils doivent avoir un souci constant d'adaptation de leur entreprise et un sens exceptionnel de la prévision. Alors seulement ils pourront, dans une action collective de tout le patronat, initiative combien souhaitable, rationaliser autant que possible la production pour éviter des licenciements périodiques, helas! trop nombreux.

\section{B-LES TRAVAILLEURS:}

\section{RôLe ACTIF DES GROUPEMENTS OUVRIERS}

Devant le fléau du chômage, les travailleurs qui en sont les premières victimes, se sentent souvent impuissants à $\mathrm{y}$ apporter un remède. L'examen attentif de la question nous indique cependant qu'ils ont eux aussi un rôle à jouer.

Isolés ils ne peuvent rien faire. Mais en s'unissant dans de saines associations ils peuvent contribuer à résoudre efficacement le problème du chômage. En effet, au sein de leurs groupements ils peuvent élaborer des suggestions positives qu'ils chercheront à communiquer à tous ceux qui sont responsables avec eux de l'organisation de la vie économique. L'action collective des associations ouvrières peut chercher à saisir l'opinion publique de leurs légitimes revendications à condition toutefois que la vérité, la justice et la charité soient toujours respectées. Les associations éviteront la démagogie qui éloigne toujours des véritables solutions, favorise un climat malsain de lutte de classes et accroît inutilement et dangereusement l'amertume et le ressentiment. L'action des associations devra avoir comme but d'éclairer les intelligences et de stimuler les volontés dans la voie des réalisations concrètes. C'est dans ce climat que naîtra un authentique esprit de collaboration sans lequel il ne peut se trouver de solution véritable.

\section{LES OUVRIERS ET LA CONDUITE DE L'ÉCONOMIE NATIONALE}

Mais pour que cette collaboration soit possible, il faut d'abond que les travailleurs puissent assumer par l'intermédiaire de leurs associations leur part de responsabilités dans la conduite de l'économie nationale. Pie XII a souvent déploné que 
les travailleurs soient étrangers à la vie économique de leur pays; à plusieurs reprises il a réclamé pour le monde ouvrier \&son accession à l'exercice plénier de ses responsabilités 》 «avec des droits égaux à ceux des autres membres 》.

La participation des travailleurs au développement de l'économie nationale devra se fonder sur la solidarité et la communauté d'intérêts et de responsabilités de tous les participants à la vie économique.

\section{C-PATRONS ET OUVRIERS:}

\section{Collaboration nécessaire.}

Le redressement de l'économie exige en outre une loyale et efficace collaboration entre patrons et ouvriers. Dans son allocution du 7 mai 1949, Pie XII dénonçait comme kerroné et funeste en ses conséquences, le préjugé qui voit (dans les préoccupations des travailleurs et des employeurs) une opposition irréductible d'intérêts divergents. L'opposition n'est qu'apparente. Dans le domaine économique, il y a communauté d'activité et d'intérêts entre chefs d'entreprise et ouvriers... Chefs d'entreprise et ouvriers ne sont pas antagonistes inconciliables. Ils sont coopérateurs dans une oeuvre commune. Ils mangent, pour ainsi dire, à la même table, puisqu'ils vivent, en fin de compte du bénéfice net et global de l'économie nationale 8 .

Les membres de la Commission qui ant rédigé le Rapport sur les perspectives économiques du Canada insistaient eux-mêmes sur la nécessité de la collaboration des groupements économiques. Après avoir prédit que «l'avenir économique qui attend le Canada est bien de nature à soulever l'enthousiasme 》, ils nous mettent en garde contre les effets funestes d'un manque de compréhension qui pourrait exister entre patrons et ouvriers.

\section{INITIATIVES CONCRÈTES - COMMISSIONS MIXTES}

Nos Très Chers Frères, Nous souhaitons que le problème du chômage et tant d'autres questions brûlantes d'actualité deviennent l'occasion de prouver par des initiatives concrètes cette communauté supérieure d'intérêts. Ne serait-il pas souhaitable que cette solidarité qui doit unir tous les agents de la vie économique setraduise pour la création de commissions mixtes, groupant les dirigeants ouvriers et patronaux, dans une entreprise d'étude en commun et d'action concertée. De tels organismes pourraient alors rechercher les moyens efficaces d'atténuer la crise actuelle, présenter des plans concrets et obtenir de l'Etat l'appui et les services jugés nécessaires. Seule une telle initiative de collaboration patronale-ouvrière, dans un esprit de co-responsabilité avec l'Etat, pourra prémunir notre vie économique des abus soit d'un libéralisme effréné qui conduit à l'anarchie, soit d'un étatisme qui opprime.

\section{D-ROLE DE L'ETAT:}

Enfin, Nos Très Chers Frères, est-il nécessaire de préciser que l'oeuvre d'assainissement de la vie économique et la lutte contre le chômage requièrent non seule- 
ment l'effort conjoint des patrons et des travailleurs mais exigent également la collaboration de l'ensemble des corps intermédiaires avec l'Etat. mission.

En effet, l'Etat, en cette matière, doit jouer un rôle positif et remplir toute sa

Il appartient d'abord aux individus et aux groupes intermédiaires de prendre toutes leurs responsabilités. Mais comme il est évident qu'en cette matière, malgré toute leur ingéniosité, ils ne pourront seuls résoudre le problème du chômage, les pouvoirs publics doivent intervenir pour stimuler leur initiative et compléter leur action. Quand donc linitiative privée reste inopérante ou insuffisante, nous rappelle le Souverain Pontife, eles pouvoirs publics sont obligés, dans la phus grande mesure possible, de procurer de l'occupation en entreprenant des travaux d'utilité générale et de faciliter, par des conseils et d'autres moyens, l'embauchage pour ceux qui le cherchent $\gg$. (Pie XII, 24 décembre 1952).

Quelques mois aurapavant S.S. Pie XII avait précisé les fonctions de l'Etat dans la production dans ces termes: «Tout d'abord le devoir d'accroître la production et de la proportionner sagement aux besoins et à la dignité de l'homme pose au premier plan la question de l'ordonnance de l'économie sur le chapitre de la production. Or, sans substituer leur omnipotence oppressive à la légitime autonomie des initiatives privées, les pouvoirs publics ont ici un rôle indéniable de coordination, qui s'impose plus encore dans l'enchevêtrement des conditions actuelles surtout sociales.

En particulier, ce n'est pas sans leur concours que peut se constituer une politique économique d'ensemble qui favorise l'active coopération de tous et l'accroissement de la production des entreprises, sounce directe de revenu national $\gg$. (Pie XII, Lettre à M. Charles Flory, pour la Semaine sociale de Dijon, 1952).

\section{DEUXIEME PARTIE}

\section{Nos responsabilités chrétiennes}

Nos Très Chers Frères, voilà donc précisés les principes de solution indispensables, du point de vue moral et social, et la lounde responsabilité des agents de l'économie.

Et maintenant, dans la seconde partie de cette lettre, Nous allons voir quelles actions chacun doit s'imposer immédiatement, s'il veut prouver réellement son sens social chrétien. A Notre avis, nous avons tous quatre devoirs d̀ remplir: à savoir celui de prier, d'examiner notre conscience, de faire la charité et enfin de poser des actes concrets si minimes soient-ils.

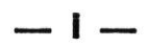

\section{Un premier devoir: prier}

Nous vous demandons d'abord, bien chers diocésains, de beaucoup prier pour que cesse cette crise de chômage, cause de tant de souffrances physiques et morales. Dans un vaste mouvement de solidarité chrétienne, demandons à Dieu de donner 
le courage et la clairvoyance à tous ceux qui, à un titre quelconque, peuvent faire quelque chose pour détendre la situation actuelle. Demandons-Lui également d'acconder à tous nos frères privés de travail et à leurs familles éprouvées, les secours matériels et spirituels dont ils ont tant besoin. Il est vrai que les périodes de crise sont parfois celles où l'on a le moins envie de prier mais, n'est-ce pas alors qu'il faudrait prier davantage? Au milieu des épreuves, la ferveur sensible fait souvent défaut. Rappelez-vous qu'elle n'est pas essentielle! Soyons-en détachés, Mes Frères, et demandons instamment à Dieu de vaincre cette grande épreuve.

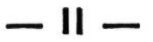

\section{En second lieu, un sérieux examen de conscience s'impose}

Le rôle de l'Eglise, en matière économique et sociale, Nous l'avons déjà dit au début de cette lettre, n'est pas de proposer des solutions techniques. Elle a pour mission avant tout d'éclairer les consciences.

C'est ainsi que Nous avons voulu, par cette Lettre Pastorale, semer en vos oonsciences une inquiétude salutaire en vous rappelant les exigences de la doctrine sociale de l'Eglise.

Nous vous invitons donc maintenant à vous poser chacun la question suivante: « Pour ètre fidèle aux requêtes de ma foi, que puis-ie faire, pour ma part, actuellement, en face de ce grave problème? services :

Si ie suis chef d'entreprise, dans lindustrie, le commerce, les finances ou les

-Ai-je fait moralement tous les efforts possibles pour éviter certains licenciements?

- Ne serais-je pas en mesure maintenant de reprendre quelques-uns de mes employés sans travail?

- Suis-je suffisamment convaincu que j'ai un devoir de justice sociale d'investir pour favoriser cette économie de progrès qui a besoin de se renouveler et de se développer constamment?

- Comme membre de diverses associations patronales, est-ce que j'ai exigé de mes représentants qu'ils passent à l'action et favorisent un effort collectif du patronat? Seule une telle action d'ensemble permettra d'étudier et de réaliser les évolutions et les améliorations nécessaires dans la production et la distribution des biens.

- Suis-je suffisamment convaincu de la collaboration qui doit exister avec les dirigeants ouvriers?

-Qu'ai-je fait pour favoriser de telles rencontres?

Si je suis travailleur ou employé :

-Ai-je bien conscience de mon devoir de contribuer de tout l'effort possible à cette économie d'expansion?

- Suis-je bien convaincu que le travailleur ou l'employé a le devoir de \& fournir intégralement et fidèlement tout le travail auquel il s'est engagé par 
contrat libre et conforme à l'équité »? (Léon XIII dans Rerum Novarum). -Et dans cette période de chômage, par solidarité avec mes camarades chômeurs, suis-je convaincu que je dois, en certains cas, éviter de cumuler les emplois?

-Ouvrier syndiqué, est-ce que j’exige de mes représentants d'être en toute première ligne de cet effort commun?

-Est-ce que je souhaite que patrons et ouvriers participent à des études conjointes sur les causes et les remèdes au chômage?

Si je suis représentant du peuple, conseiller, député ou ministre, dans l'une ou lautre administration gouvernementale :

-Suis-je bien conscient des lourdes responsabilités de mon mandat face au problème actuel du chômage?

-Suis-je bien convaincu que l'Etat lui-même a l'importante mission de prévenir les troubles de l'équilibre économique résultant tant de la multiplicité et des conflits des égoïsmes opposés, individuels et collectifs? 》 (Pie XII, ler juin 1941):

《 Que l'Etat doit protéger le dnoit au travail des citoyens (non pas qu'en dehors d'une société collectiviste, il ait à fournir lui-même un emploi), mais il doit veiller à réduire les risques du chômage et à en faire compenser les inconvénients par l'influence qu'il pourra exercer sur les organismes économiques et professionnels dont il aura soin cependant de respecter l'autonomie »? (NN.SS. les archevêques et évêques de France, Directoire no 116).

$\rightarrow$ Serviteur du bien commun, ai-je fait tout mon possible à ce jour pour empêcher ou adoucir ces souffrances humaines?

Enfin, Nos Très Chers Frères, dans cet examen de conscience que nous devons tous faire, en cette heure de crise, nous ne pouvons pas ne pas déplorer l'accroissement intolérable de certaines dépenses de luxe, des dépenses superflues et déraisonables qui contrastent durement avec la misère d'un grand nombre d'économiquement faibles. Nous savons bien qu'en termes économiques, ces dépenses permettent aux uns et aux autres de gagner leur vie. Mais, demandons-nous bien si ces sommes fabuleuses ne pourraient pas être investies et cette main-d'oeuvre employée à des firs socialement et moralement plus utiles? Nous savons, par exemple, que certains objets de consommation dont vous faites un usage immodéré, permettent au président de cette compagnie de dépenser plusieurs millions de dollars, chaque hiver, sur la Riviera française...

Pie XII ne déplorait-il pas que «...t tant de richesses forment ou se perdent dans le gaspillage, mais qui remises en circulation, pourraient concourir, par un emploi judicieux et profitable, au bien-être de tant de familles...»? (Pie XII, 5 juillet 1952).

\section{$-\mathrm{III}-$}

\section{Un autre devoir: celui de faire la charité}

Il n'y a pas que les rochers qui subissent la douloureuse érosion du temps. Les mots connaissent, eux aussi, la lente dégradation à quoi rien ne résiste. A force d'en user, on les use! 
A cette loi de l'usure, l'expression \& faire la charité » n'a pas échappé; elle s'est vidée peu à peu de sa richesse première. A un tel point qu'il nous faut faire effort aujourd'hui pour atteindre l'essentiel de cette vérité.

La gratuité de la charité chrétienne a été la première victime de cette dégradation. Loin d'être un geste d'entr'aide tout spontané, l'aumône est muée trop souvent, pour plusieurs, en une mesure de protection. On donne au pauvre comme à une société d'assurance ou comme à un guichet de banque.

Nos Très Chers Frères, Nous vous exhortons à retrouver le sens authentique de l'aumône.

L'aumône est un acte de religion. Elle est aussi un acte de pénitence et de mortification. Mais elle est bien autre chose encore. Elle est une charité, une bienveillanoe. Son objet n'est pas seulenent d'honorer Dieu et de s'amender soi-même, mais aussi de subvenir aux besoins du prochain.

Nous vous engageons donc, bien chers Frères, selon l'exhortation du prophète Isaïe, à rompre votre pain avec celui qui a faim. (Is. 48, 7).

Que tous voient dans tout chômeur un frère qui souffre. Qu'ils s'intendisent d'abond tout jugement blessant et injuste et qu'ils s'efforcent de secourir et d'aider ceux qui sont actuellement privés de revenus.

Autour de vous, au nombre de vos parents et de vos amis, dans bien des milieux que vous connaissez, il y a sûrement des familles qui souffrent. Allez vers. elles!

L'organisation officielle d'assurances sociales ne dispense nullement du devoir d'exercer individuellement l'aumône chrétienne. Les allocations d'assistance ne représentent qu'un strict minimum, nettement insuffisant dans la plupart des cas. Nombre de victimes du chômage, pour diverses raisons techniques, ne reçoivent pas d'allocations. XII, 26).

«Quand un membre souffre, dit saint Paul, tout le corps souffre. (I Cor.

C'est donc pour nous tous un strict devoir de donner loyalement notre concours généreux à toute organisation officielle ou privée qui se propose de venir en aide aux victimes du chômage.

\section{- IV -}

\section{Enfin, une action symbolique}

Voulant joindre à cette exhortation pastorale l'éloquence de l'exemple, Nous avons décidé, bien chers diocésains, non sans avoir consulté nos collaborateurs, d'ouvrir immédiatement quelques chantiers de construction. Par ce geste, Nous désirons d'abord procurer du pain à nos frères qui n'en ont pas, mais aussi, Nous voulons poser un acte susceptible d'entraîner d'autres initiatives de nos concitoyens.

L'ouverture de ces chantiers sera bien loin de régler lactuelle crise de chômage! Il ne s'agit donc, dins notre intention et dans la réalité, que d'une modeste action symbolique. 
Pour ce faire, Nous tirons pour ainsi dire, une traite sur la Providence et comptons sur votre coutumière générosité. Des quêtes spéciales auront lieu tous les mois dans les paroisses. Elles seront annoncées par Messieurs les Curés qui les organiseront selon le mode qu'ils auront décidé.

Les fruits de ces collectes Nous seront envoyés fidèlement et personnellement chaque mois. Nous vous ferons connaître les résultats obtenus et Nous vous parlerons des chantiers que Nous aurons ouverts.

Vous êtes généreux lorsque les missionnaires vous tendent la main. L'oeuvre que Nous vous recommandons aujourd'hui mérite votre bienveillance.

Nous faisons un appel pressant à ceux qui ont des sommes d'angent non investies et non soumises au contrôle de l'impôt. En vensant une aumône pour « Nos chantiers », vous nétablirez l'équilibre social que la cupidité avait rompu. Les sommes que vous déciderez de verser à cette oeuvre doivent Nous être adressées personnellement.

L'aumône enrichit au ciel celui qui s'appauvrit sur terre. Au chapitre 12ème de Saint Luc, nous lisons le conseil suivant: \& Vendez vos biens et donnez-les en aumônes. Faites-vous des bourses qui ne s'usent pas, un trésor qui ne vous fera pas défaut dans les cieux, où ni voleur n'approche, ni mite ne détruit. Car où est votre trésor, là aussi sera votre coeur $\gg$. (Luc 12, 33-34).

Bien Chers Frères, tout l'argent ainsi recueilli sera remis en circulation immédiate en salaires et achats de matériaux. Ainsi permettra-t-il à des concitoyens de connaitre dès demain, un peu plus de sécurité.

Comme il serait encourageant de voir des entreprises industrielles et commerciales, des fabriques paroissiales, des communautés religieuses et différents organismes civils, faire diligence pour mettre en chantier sans tarder, certains des travaux qu'ils ont déjà projetés. Il en coûtera évidemment certains sacrifices, mais quelle espérance ne feront-ils pas naître dans notre milieu!

\section{Conclusion}

Et Nous devons maintenant conclure.

Notre ultime appel sera pour une action responsable et efficace de tous Nos diocésains.

Trop de chrétiens ont malheureusement tendance à croire que lorsqu'ils n'ont pas positivement posé d'actes répréhensibles, leur conscience est en ordre; ils ont l'impression d'avoir fait ainsi tout leur devoir.

Nos Très Chers Frères, nous devons être des citoyens vraiment actifs dans le Royaume de Dieu. Nous avons la stricte obligation de faire le bien. Or, ce peut être parfois un grave péché que de ne pas travailler à néaliser effectivement autour de soi l'ondre social chrétien.

Nous devons regretter l'individualisme de beaucoup de gens honnêtes qui privent les groupements et les oeuvres de leur présence, de leur appui et de l'esprit de justice et de charité qu'ils pourraient y faire rayonner. 
Dans un régime démocratique, chacun a un rôle authentique à jouer, tantôt par son vote ou son influence, tantôt par son action et ses oeuvres.

Soyons donc, Bien Chers Frères, d'authentiques disciples du Christ; que par une politique de présence et d'action, nous soyons en tout temps et partout les témoins et les apôtres de la doctrine sociale de l'Eglise.

Sous l'éclairage de la justice, de la charité et de la vérité, elle nous fait si bien découvrir toutes les exigences actuelles du bien commun en face du chômage.

En souhaitant que la noblesse de l'entreprise vous attire et son ungence vous stimule, en priant Dieu d'inspirer vos efforts et d'adoucir les souffrances matérielles et spirituelles, Nous vous accondons affectueusement Notre paternelle bénédiction.

Donné à Montréal, le quatorze janvier, en la fête de Saint-Hilaire, en l'an du Seigneur mil neuf cent cinquante-neuf.

\title{
UNEMPLOYMENT AND OUR RESPONSIBILITIES AS CHRISTIANS
}

\author{
Cardinal Paul-Emie Léger
}

1st PART

The Actual Situation and the Elements
of a Just and Lasting Solution

$-1-$

\section{The Gravity of the Actual Situation}

During the past few years, the plague of unemployment has affected our society with persistence and in a steadily increasing degree. This evil afflicts a very large number of workers, brings misery to their families and seriously jeopardizes social order.

We have been told that there are more than 80,000 unemployed in Montreal; if we consider that each unemployed worker is responsible for the sustenance of an average of two other persons, we must conclude that there are about 240,000 persons who are victims of unemployment.

We have a more precise idea of the gravity of the situation when we realize that 10 to 15,000 of the unemployed are young. These young men come to society with all their vigour, their legitimate hopes and their will to work, and unfortunately find that no employment is offered to them.

Who would dare to say, in the face of this situation, that all types of unemployment are normal and, at the most, constitute a "necessary evil"? Such a statement would be as little in agreement with the natural law as it is with 